\title{
Elevated levels of erythrocyte- conjugated dienes indicate increased lipid peroxidation in schistosomiasis mansoni patients
}

H.T.F. Facundo ${ }^{1}$, C.T. Brandt ${ }^{2}$, J.S. Owen ${ }^{3}$ and V.L.M. Lima ${ }^{1}$

\author{
1Departamento de Bioquímica, Centro de Ciências Biológicas, and \\ ${ }^{2}$ Departamento de Cirurgia, Hospital das Clínicas, \\ Universidade Federal de Pernambuco, Recife, PE, Brasil \\ ${ }^{3}$ Royal Free Hospital and University College Medical School, London, UK
}

\section{Correspondence \\ V.L.M. Lima \\ Departamento de Bioquímica \\ CCB, UFPE \\ Av. Prof. Moraes Rego, $\mathrm{s} / \mathrm{n}$ \\ 50670-420 Recife, PE \\ Brasil \\ Fax: +55-81-2126-8541 \\ E-mail: vlml@ufpe.br}

Presented at the XVII Annual

Meeting of the Federação de Sociedades de Biologia Experimental, Salvador, BA, Brazil, August 28-31, 2002.

Research supported by CNPq (No. 521340/96-9), BNB (No. 007-98) and CTPETRO-FINEP (No. 65.99.0485.00).

Received October 30, 2003 Accepted April 5, 2004

\begin{abstract}
Schistosoma mansoni causes liver disease by inducing granulomatous inflammation. This favors formation of reactive oxygen species, including superoxide ions, hydrogen peroxide and hydroxyl radicals all of which may induce lipid peroxidation. We have evaluated lipid peroxidation in 18 patients with hepatosplenic schistosomiasis mansoni previously treated with oxamniquine followed by splenectomy, ligature of the left gastric vein and auto-implantation of spleen tissue, by measuring levels of erythrocyte-conjugated dienes and plasma malondialdehyde (MDA). Age-matched, healthy individuals $(\mathrm{N}=18)$ formed the control group. Erythrocyteconjugated dienes were extracted with dichloromethane/methanol and quantified by UV spectrophotometry, while plasma MDA was measured by reaction with thiobarbituric acid. Patient erythrocytes contained two times more conjugated dienes than control cells (584.5 \pm 67.8 vs $271.7 \pm 20.1 \mu \mathrm{mol} / 1, \mathrm{P}<0.001)$, whereas the increase in plasma MDA concentration (about 10\%) was not statistically significant. These elevated conjugated dienes in patients infected by $S$. mansoni suggest increased lipid peroxidation in cell membranes, although this was not evident when a common marker of oxidative stress, plasma MDA, was measured. Nevertheless, these two markers of lipid peroxidation, circulating MDA and erythrocyte-conjugated dienes, correlated significantly in both patient $(\mathrm{r}=$ $0.62 ; \mathrm{P}<0.01)$ and control $(\mathrm{r}=0.57 ; \mathrm{P}<0.05)$ groups. Our data show that patients with schistosomiasis have abnormal lipid peroxidation, with elevated erythrocyte-conjugated dienes implying dysfunctional cell membranes, and also imply that this may be attenuated by the redox capacity of antioxidant agents, which prevent accumulation of plasma MDA.
\end{abstract}

Key words

- Lipid peroxidation

- Conjugated dienes

- Malondialdehyde

- Schistosomiasis mansoni 
Schistosomiasis mansoni is endemic in northeast Brazil, mainly in rural areas of Pernambuco. The disease is also extending to new areas, for example the vacation island of Itamaracá, $50 \mathrm{~km}$ to the north of Recife (1), as infected people migrate. Patients with severe infections frequently develop periportal fibrosis, portal hypertension and hepatosplenomegaly and, classically, are surgically treated by splenectomy, accompanied by obliterating suture of esophageal varices and ligature of the left gastric vein (2). In the last decade, auto-implantation of spleen tissue in children has been carried out with the surgery and this has improved liver function and increased survival time (3). Among tropical diseases, Schistosoma mansoni is the second major cause of morbidity and mortality worldwide (2) and persists mainly in developing countries, with significant economic and public health consequences (1). Morbidity depends on genetic and environmental factors, as well as severity of infection, and all three influence the granulomatous inflammation of the liver and later fibrosis around the eggs (2).

Eosinophil cells associated with schistosome-induced granulomas form oxygen free radicals, such as superoxide and hydroxyl radicals (4), and release active eosinophil peroxidase around the egg granulomas (5). However, the consequences of free radical generation in schistosomiasis mansoni are still unknown. This also applies to the effects on lipid peroxidation, where polyunsaturated fatty acids and other lipids are oxidized by intermediate free radicals to form conjugated dienes, malondialdehyde (MDA) and lipid hydroperoxides, among other products (6). MDA is the most abundant aldehyde produced during lipid peroxidation, and its assay is often used as a marker for oxidative stress in several diseases (7), including Alzheimer's disease, atherosclerosis and diabetes (8). In liver disease, free radicals have been implicated in the inflammatory process, and increased lipid peroxidation has been found
(9). Lipid peroxidation starts with abstraction of $\mathrm{OH}^{\bullet}$ from a $-\mathrm{CH}_{2}$ - group of polyunsaturated fatty acids, where the carbon radical is usually stabilized by a molecular rearrangement forming conjugated dienes, compounds containing two double bonds separated by a single bond. Conjugated dienes react with $\mathrm{O}_{2}$ forming peroxyl radicals that react with $\mathrm{OH}^{\bullet}$ atoms from other lipids, producing lipid hydroperoxides or forming cyclic peroxides, and several products are formed, including MDA (6). In mice, the antioxidant capacity of livers damaged by $S$. mansoni is reduced, and this results in generation of lipid peroxides (10).

The aim of the present study was to evaluate lipid peroxidation in patients with schistosomiasis mansoni who were submitted to clinical and surgical treatment, measuring levels of plasma MDA and erythrocyteconjugated dienes.

Thiobarbituric acid, MDA and methanol were obtained from Sigma (St. Louis, MO, USA) and dichloromethane and trichloroacetic acid from Vetec (Rio de Janeiro, RJ, Brazil). All other solvents and chemicals were of analytical grade.

Young patients (11-20 years; $\mathrm{N}=18)$ of both genders with hepatosplenic schistosomiasis mansoni, who had had upper digestive bleeding but no renal, cardiac, hepatitis, or other parasite/microbial associated disease were included in this study. They were outpatients at the Clinical Hospital, Federal University of Pernambuco (UFPE), Recife, and had been treated with the antischistosomal drug, oxamniquine (a single dose of $20 \mathrm{mg}$ / $\mathrm{kg}$ ) followed by splenectomy, ligature of the left gastric vein and auto-implantation of spleen tissue into an omental pouch of the major omentum. The study was conducted on patients 3 to 6 years after the surgical procedure. Patients had generally developed normally, although development improved after treatment, as did their indicators of liver function, especially serum albumin concentration and prothrombin time (3). Body mass 
index (BMI) was calculated as the body weight $(\mathrm{kg})$ per height ${ }^{2}\left(\mathrm{~m}^{2}\right)$. None of the women in either group were pregnant or used oral contraceptives. The study was approved by the Ethics Committee of the University Hospital, UFPE (Process number 193/99-CEP/CCS) and written informed consent was obtained from all patients and volunteers used as controls.

Early morning blood samples were collected into ice-cold tubes containing EDTA $(1 \mathrm{mg} / \mathrm{ml})$ and erythrocytes and plasma were isolated by centrifugation at 3,000 $\mathrm{g}$ for 15 min at $4^{\circ} \mathrm{C}$. As storage at $-20^{\circ} \mathrm{C}$ may increase lipid peroxidation, duplicate samples were analyzed immediately. Plasma thiobarbituric acid reactive substance (TBARS) were measured by the method of Buege and Aust (11), mixing aliquots with $15 \%(\mathrm{w} / \mathrm{v})$ trichloroacetic acid and $0.375 \%(\mathrm{w} / \mathrm{v})$ thiobarbituric acid and heating at $100^{\circ} \mathrm{C}$ for $15 \mathrm{~min}$. Samples were cooled to room temperature, centrifuged at 3,000 $\mathrm{g}$ for $5 \mathrm{~min}$ and their absorbance was measured at $535 \mathrm{~nm}$ against a reaction mixture lacking plasma but subjected to the same procedure. As in other studies (12), levels of TBARS are reported as nmol of MDA per liter of plasma, using a standard curve prepared with MDA bis (diethyl acetal) as the MDA reference source. Conjugated dienes in washed erythrocytes were extracted with dichloromethane/methanol $(2: 1, v / v)$ and measured by the method of Buege and Aust (11). A solution of $50 \mathrm{mM}$ potassium chloride was then added and, after the preparation was left to stand overnight at $4^{\circ} \mathrm{C}$, the concentration of conjugated dienes in the lower phase was measured by absorbance at $233 \mathrm{~nm}$ against dichloromethane (11).

Results were analyzed by the unpaired Student $t$-test and Pearson's correlation coefficient; both were calculated using the Origin software program version 5.0, and differences were considered significant for $\mathrm{P}<0.05$. Data in the text are reported as means \pm SEM. No gender-related difference was observed in the correlation analysis and so data for both genders were used as a single group.

Most studies exclude patients considered to be malnourished, usually based on BMI scores or on plasma protein and hemoglobin levels. Here, we used BMI and dietary history to exclude malnourished patients. All patients included lived at home with their mother or a caregiver responsible for preparing food, and no significant changes in their dietary habits were noted as a result of the disease. In addition, the BMI of our patients (19.3 \pm $1.2 \mathrm{~kg} / \mathrm{m}^{2}$ ) did not differ from the control group $\left(21.7 \pm 0.5 \mathrm{~kg} / \mathrm{m}^{2} ; \mathrm{P}>0.05\right)$.

One indicator of lipid peroxidation, plasma MDA concentration, was increased by $10 \%$ in patients with schistosomiasis mansoni (Figure 1A), but the rise was not significant. However, levels of conjugated dienes extracted from patient erythrocytes were significantly increased by about $100 \%(\mathrm{P}<0.001)$ in comparison to the control group (Figure 1B). Moreover, these two markers of lipid peroxidation, circulating MDA and erythrocyte-conjugated dienes, were significantly correlated in both patient $(\mathrm{r}=0.62 ; \mathrm{P}<0.01)$ and control $(\mathrm{r}=0.57 ; \mathrm{P}<0.05)$ groups (Figure 2).

The clinical manifestations of schistoso-

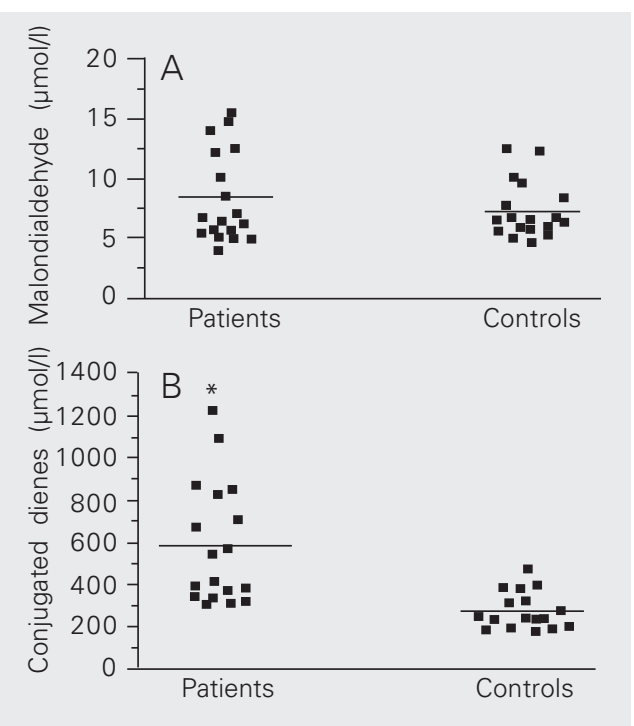

Figure 1. Concentration of lipid oxidation products in plasma and erythrocytes of patients with hepatosplenic schistosomiasis mansoni. Plasma malondialdehyde (A) and erythrocyteconjugated diene (B) levels in 18 patients with schistosomiasis, who had been treated by splenectomy, obliterative suture of the esophageal varices, ligature of the left gastric vein, and auto-implantation of spleen tissue. ${ }^{*} P<0.05$ compared to controls (unpaired Student ttest). 
Figure 2. Correlation between plasma malondialdehyde and erythrocyte-conjugated dienes. $A$, Patients with schistosomiasis mansoni. $B$, Healthy individuals (control group). Data were analyzed by the Pearson correlation method. miasis range from the mild intestinal form to severe hepatosplenic forms associated with esophageal varicose veins and upper digestive bleeding. Infection caused by S. mansoni can induce granulomatous inflammation of the liver and lead to formation of certain reactive oxygen species, such as superoxide ions $\left(\mathrm{O}_{2}{ }^{--}\right)$and hydroxyl radicals $\left(\mathrm{OH}^{\bullet}\right)$. These promote lipid peroxidation (4), an adverse event which contributes to the pathology associated with schistosomiasis. Lipid peroxidation is a complex and relatively imprecise series of reactions leading to a diverse array of bioactive molecules, many still ill-defined (6). Perhaps not surprisingly, given its complexity, there is no clear agreement on how best to quantify lipid peroxidation and the techniques used range from relatively crude measures to more sophisticated analyses of individual products using HPLC and/or mass spectrometry. As our study is the first investigation of lipid peroxidation in patients with schistosomiasis, we

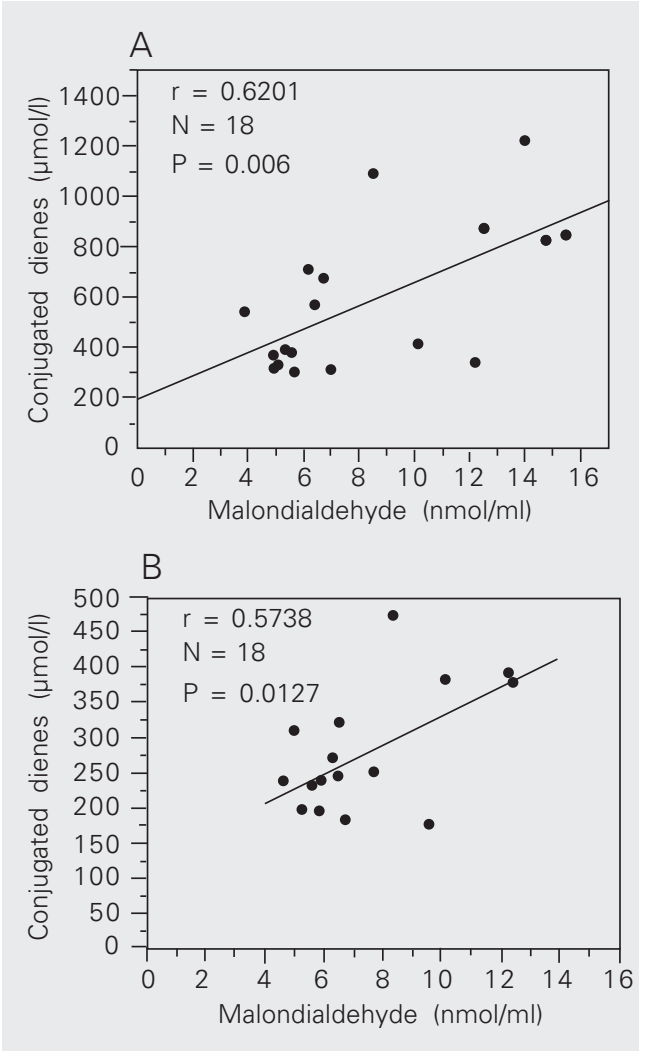

chose two practical assays for oxidative stress, i.e., plasma TBARS and erythrocyteconjugated dienes. These markers correlate closely with other methods commonly used to detect lipid peroxidation and are wellcharacterized and reliable indicators $(7,13)$. However, while the TBARS assay is frequently used as a measure of MDA, with data expressed as nmol MDA per liter plasma $(12,14)$ as we did in the present study, the assay is not specific for MDA since other compounds including sugars, amino acids and bilirubin may cross-react with thiobarbituric acid. Thus, in future studies it may be preferable to use HPLC, a more precise though complex analysis (14).

In the present study, we found clear evidence of lipid peroxidation in erythrocytes from patients with schistosomiasis mansoni, who had undergone clinical and surgical treatment, as judged by their significantly high content of conjugated dienes, products which reflect the initial phase of lipid peroxidation. On the other hand, when the degradative phase of lipid peroxidation was examined in the plasma of these patients, assaying TBARS as a measure of MDA levels, there was no significant increase compared to normal subjects.

The increased lipid peroxidation in patient erythrocytes seems to be a consequence of the disease rather than of clinical treatment with oxamniquine. Indeed, plasma MDA levels were not altered in $S$. mansoni-infected mice treated with praziquantel, a drug used to kill the parasite (15). Furthermore, our patients did not have granulomatous inflammation, which is known to increase lipid peroxidation and raise levels of conjugated dienes (4). However, our patients do still have moderate liver disease, and in a study of mild alcohol-induced liver damage lipid peroxidation was detected by various indicators of oxidative stress, even though plasma MDA levels were normal (16).

Erythrocytes constitute a well-established model to study cytotoxic damage to mem- 
branes by chemical and physical free radical promoters. As shown here, schistosomiasis mansoni may induce chemical damage to erythrocyte membranes through an oxidative stress pathway. Superoxide anion, singlet oxygen and $\mathrm{H}_{2} \mathrm{O}_{2}$ can all contribute to erythrocyte lipid oxidation with the formation of intermediate conjugated dienes preceding that of aldehyde products (TBARS). The reactions begin when the main endogenous lipophilic antioxidant, vitamin E, is extensively consumed and in experimental schistosomiasis mansoni vitamin E supplementation lowers the activity of catalase and glutathione peroxidase, liver enzymes involved in antioxidant mechanisms (17). In the present study, schistosomiasis appeared to promote lipid peroxidation, as measured by increased erythrocyte-conjugated diene concentration. However, excessive plasma MDA was not produced, perhaps because reduced glutathione (GSH) and protein thiols were preserved, although, as yet, the redox capacity of GSH/oxidized glutathione (GSSG) and antioxidant levels of vitamin $\mathrm{E}$ and $\mathrm{C}$ have not been measured in human schistosomiasis. Thus, although we have examined two blood markers of oxidative stress in this preliminary study of patients with schistosomiasis, it will be important in future studies to determine how changes in lipid peroxidation markers relate to the disease process and to reductions in antioxidant defenses, including the consumption of ascorbate and other antioxidant nutrients during free radical scavenging. For example, in elderly people blood antioxidant defenses are significantly reduced, and in patients with unstable hemoglobin disorders or low levels of glucose-6-phosphate dehydrogenase the erythrocytes are susceptible to oxidative attack, predisposing them to drug- or infection-mediated hemolytic crises. Furthermore, when hepatic granulomas develop in experimental schistosomiasis they trigger production of reactive oxygen species which alter the antioxidant defense profile (5).

Our finding of a positive correlation between plasma MDA and erythrocyte-conjugated dienes supports the use of these routine and practical assays as a means of identifying and monitoring patients susceptible to severe lipid peroxidation in schistosomiasis. The correlation is also consistent with a mechanism of a reactive oxygen species-induced lipid peroxidation process in which polyunsaturated fatty acids undergo hydrogen abstraction by free radical attack to form conjugated dienes $(6,9)$, which are further attacked to generate MDA or lipid hydroperoxide. Thus, conjugated dienes are intermediates during MDA production and this could explain the positive and significant correlation between them, both in patients and control groups. However, we recently reported a significant reduction in activity of lecithin-cholesterol acyltransferase, the plasma enzyme which esterifies cholesterol and helps regulate cell membrane lipid composition, in patients with schistosomiasis who had undergone the same clinical and surgical treatments as the patients in the present study (18). This thiol-containing enzyme is highly sensitive to several oxidizing species, either directly or by crosslinking of its co-factor, apoAI (19), and so oxidative stress may contribute to the acquired lecithin-cholesterol acyltransferase deficiency and membrane disturbances (20) seen in human schistosomiasis.

\section{References}

1. Barbosa CS, Montenegro SML, Abath FGC \& Domingues ALC (2001). Specific situations related to acute schistosomiasis in Pernambuco, Brazil. Memórias do Instituto Oswaldo Cruz, 96: 169172 .
2. Ouma JH, Vennervald BJ, Kariuki HC \& Butterworth AE (2001). Morbidity in schistosomiasis: an update. Trends in Parasitology, 17: 117-118.

3. Brandt CT, Maciel DSTM \& Caneca OAF (1997). Splenectomy 
associated with ligature of the left gastric vein in children with surgical schistosomiasis: analyses of the hepatic functional reserve. Acta Cirúrgica Brasileira, 12: 150-153.

4. McCormick ML, Metwali A, Railsback MA, Weinstock JV \& Britigan BE (1996). Eosinophils from schistosome-induced hepatic granulomas produce superoxide and hydroxyl radical. Journal of Immunology, 157: 5009-5015.

5. Gharib B, Abdallahi OMS, Dessein H \& de Reggi M (1999). Development of eosinophil peroxidase activity and concomitant alteration of the antioxidant defenses in the liver of mice infected with Schistosoma mansoni. Journal of Hepatology, 30: 594-602.

6. Halliwell B \& Gutteridge JMC (1999). Free Radicals in Biology and Medicine. 3rd edn. Oxford University Press, Oxford, UK.

7. Lefèvre G, Beljean Leymarie M, Beyerle F, Bonnefont Rousselot D, Cristol JP, Thérond P \& Torreilles J (1998). Evaluation of lipid peroxidation by measuring thiobarbituric acid reactive substances. Annales de Biologie Clinique, 56: 305-319.

8. Stohs SJ (1995). The role of free radicals in toxicity and disease. Journal of Basic and Clinical Physiology and Pharmacology, 6: 205228.

9. Gaté L, Paul J, Nguyen B, Tew KD \& Tapiero H (1999). Oxidative stress induced in pathologies: the role of antioxidants. Biomedicine and Pharmacotherapy, 53: 169-180.

10. Abdallahi OMS, Hanna S, de Reggi M \& Gharib B (1999). Visualization of oxygen radical production in mouse liver in response to infection with Schistosoma mansoni. Liver, 19: 495-500.

11. Buege JA \& Aust SD (1978). Microsomal lipid peroxidation. Methods in Enzymology, 52: 302-310.

12. Neiva TJC, Benedetti AL, Tanaka SMCN, Santos JI \& D'Amico EA (2002). Determination of serum aluminum, platelet aggregation and lipid peroxidation in hemodialyzed patients. Brazilian Journal of Medical and Biological Research, 35: 345-350.

13. Pompella A, Maellaro E, Casini AF, Ferrali M, Ciccoli L \& Comporti M
(1987). Measurement of lipid in vivo: a comparison of different procedures. Lipids, 22: 206-211.

14. de Zwart LL, Meerman JHN, Commandeur JNM \& Vermeulen NPE (1999). Biomarkers of free radical damage applications in experimental animals and in humans. Free Radical Biology and Medicine, 26: 202-226.

15. Shaheen AA, Elfattah A \& Ebeid FA (1994). Effect of praziquante treatment on lipid peroxide levels and superoxide-dismutase activity in tissues of healthy and Schistosoma mansoni-infected mice. Arzneimittel-Forschung (Drug Research), 44: 94-96.

16. Punchard NA, Senturk H, Teare JP \& Thompson RP (1994). Resistance of erythrocytes to lipid peroxidation in alcoholic patients. Gut, 35: 1753-1756.

17. Silva CA, Ferraz FH, Souza DS, Mendes AVM, Lima VLM \& Chaves MEC (2001). Effect of vitamin $E$ on hepatic enzymes in mice infected with Schistosoma mansoni. Bioavailability and biological activity, Bl-15:157-158. Association de Pharmacie Galénique Industrielle - APGI, Paris, France. VI Pharmatech: III Annual Meeting of the SBTF, APGI Symposium on Membrane Transport and III Meeting of Pharmaceutical Quality Control-ENECQ, Recife, PE, Brazil, August 5-8.

18. Silva CA, Oliveira KF, Carvalho VCO, Domingues ALC, Brandt CT \& Lima VLM (2001). Efeito de tratamento cirúrgico sobre a atividade da enzima hepática lecitina:colesterol aciltransferase (LCAT) na esquistosomose mansônica. Acta Cirúrgica Brasileira, 17 (Suppl 1): 28-30.

19. Bielicki JK, Forte TM \& McCall MR (1995). Minimally oxidized LDL is a potent inhibitor of lecithin:cholesterol acyltransferase activity. Journal of Lipid Research, 37: 1012-1021.

20. Lima VL, Sena VL, Stewart B, Owen JS \& Dolphin PJ (1998). An evaluation of the marmoset Callithrix jacchus (sagui) as an experimental model for the dyslipoproteinemia of human schistosomiasis mansoni. Biochimica et Biophysica Acta, 1393: 235-243. 